\title{
Lyapunov-Based Exponential Tracking Control of a Hypersonic Aircraft with Aerothermoelastic Effects
}

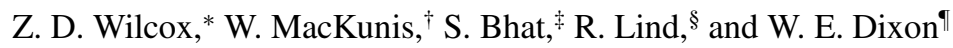 \\ University of Florida, Gainesville, Florida 32611-6250
}

DOI: $10.2514 / 1.46785$

\begin{abstract}
Hypersonic flight conditions produce temperature variations that can alter both the structural dynamics and flight dynamics. These aerothermoelastic effects are modeled by a nonlinear, temperature-dependent, parameter-varying state-space representation. The model includes an uncertain parameter-varying state matrix, an uncertain parameter-varying nonsquare (column-deficient) input matrix, and a nonlinear additive bounded disturbance. A Lyapunov-based continuous robust controller is developed that yields exponential tracking of a reference model, despite the presence of bounded nonvanishing disturbances. Simulation results for a hypersonic aircraft are provided to demonstrate the robustness and efficacy of the proposed controller.
\end{abstract}

\section{Introduction}

$\mathbf{T}$ HE design of guidance and control systems for airbreathing hypersonic vehicles (HSV) is challenging because the dynamics of the HSV are complex and highly coupled [1], and temperature-induced stiffness variations impact the structural dynamics [2]. The structural dynamics, in turn, affect the aerodynamic properties. Vibration in the forward fuselage changes the apparent turn angle of the flow, which results in changes in the pressure distribution over the forebody of the aircraft. The resulting changes in the pressure distribution over the aircraft manifest themselves as thrust, lift, drag, and pitching-moment perturbations [1]. To develop control laws for the longitudinal dynamics of a HSV capable of compensating for these structural and aerothermoelastic effects, structural temperature variations and structural dynamics must be considered.

Aerothermoelasticity is the response of elastic structures to aerodynamic heating and loading. Aerothermoelastic effects cannot be ignored in hypersonic flight, because such effects can destabilize the HSV system [2]. A loss of stiffness induced by aerodynamic heating has been shown to potentially induce dynamic instability in supersonic/hypersonic flight speed regimes [3]. Yet active control can be used to expand the flutter boundary and convert unstable limit cycle oscillations (LCO) to stable LCO [3] $]$. An active structural controller was developed [4], which accounts for variations in the HSV structural properties resulting from aerothermoelastic effects. The control design [4] models the structural dynamics using a linearparameter-varying ( $\overline{\mathrm{LPV}}$ ) framework, and states the benefits to using the LPV framework are twofold: the dynamics can be represented as a single model, and controllers can be designed that have affine dependency on the operating parameters.

Previous publications have examined the challenges associated with the control of HSVs. For example, HSV flight controllers are

Received 18 August 2009; revision received 24 February 2010; accepted for publication 25 February 2010. Copyright () 2010 by the American Institute of Aeronautics and Astronautics, Inc. All rights reserved. Copies of this paper may be made for personal or internal use, on condition that the copier pay the $\$ 10.00$ per-copy fee to the Copyright Clearance Center, Inc., 222 Rosewood Drive, Danvers, MA 01923; include the code 0731-5090/10 and $\$ 10.00$ in correspondence with the CCC.

${ }^{*}$ Graduate Research Assistant, Mechanical and Aerospace Engineering Department; zibrus@uff.edu. Student Member AIAA.

${ }^{\dagger}$ NRC Research Associate, U.S. Air Force Research Laboratory, 101 West Eglin Boulevard, Eglin AFB, FL 32542; mackunis@ufl.edu.

${ }^{\ddagger}$ Graduate Research Assistant, Mechanical and Aerospace Engineering Department; sanketh@ufl.edu. Student Member AIAA.

${ }^{\S}$ Associate Professor, Mechanical and Aerospace Engineering Department; ricklind@ufl.edu. Associate Fellow AIAA.

${ }^{\top}$ Associate Professor, Mechanical and Aerospace Engineering Department; wdixon@ufl.edu. designed using genetic algorithms to search a design parameter space in which the nonlinear longitudinal equations of motion contain uncertain parameters [5-7]. Some of these designs use Monte Carlo simulations to estimate system robustness at each search iteration. Another approach [7] is to use fuzzy logic to control the attitude of the HSV about a single low-end flight condition. While such approaches [ [ -7 ] generate stabilizing controllers, the procedures are computationally demanding and require multiple evaluation simulations of the objective function and have large convergent times. An adaptive gain-scheduled controller [] ] was designed using estimates of the scheduled parameters, and a semi-optimal controller is developed to adaptively attain $H_{\infty}$ control performance. This controller yields uniformly bounded stability due to the effects of approximation errors and algorithmic errors in the neural networks. Feedback linearization techniques have been applied to a controloriented HSV model to design a nonlinear controller [9]. The model [9] is based on a previously developed [10] HSV longitudinal dynamic model. The control design [9] neglects variations in thrust lift parameters, altitude, and dynamic pressure. Linear output feedback tracking control methods have been developed [11], in which sensor placement strategies can be used to increase observability, or reconstruct full state information for a state-feedback controller. A robust output feedback technique is also developed for the linearparameterizable HSV model, which does not rely on state observation. A robust setpoint regulation controller [12] is designed to yield asymptotic regulation in the presence of parametric and structural uncertainty in a linear-parameterizable HSV system.

An adaptive controller [13] was designed to handle (linear in the parameters) modeling uncertainties, actuator failures, and nonminimum phase dynamics [14] for a HSV with elevator and fuel ratio inputs. Another adaptive approach [15] was recently developed with the addition of a guidance law that maintains the fuel ratio within its choking limits. While adaptive control and guidance control strategies for a HSV are investigated [13-15], neither addresses the case in which dynamics include unknown and unmodeled disturbances. There remains a need for a continuous controller, which is capable of achieving exponential tracking for a HSV dynamic model containing aerothermoelastic effects and unmodeled disturbances (i.e., nonvanishing disturbances that do not satisfy the linear in the parameters assumption).

In the context of the aforementioned literature, the contribution of the current effort (and the preliminary effort by the authors [6]) is the development of a controller that achieves exponential model reference output tracking despite an uncertain model of the HSV that includes nonvanishing exogenous disturbances. A nonlinear temperature-dependent parameter-varying state-space representation is used to capture the aerothermoelastic effects and unmodeled uncertainties in a HSV. This model includes an unknown parametervarying state matrix, an uncertain parameter-varying nonsquare 
(column-deficient) input matrix, and a nonlinear additive bounded disturbance. To achieve an exponential tracking result in light of these disturbances, a robust, continuous Lyapunov-based controller is developed that includes a novel implicit learning characteristic that compensates for the nonvanishing exogenous disturbance. That is, the use of the implicit learning method enables the first exponential tracking result by a continuous controller in the presence of the bounded nonvanishing exogenous disturbance. To illustrate the performance of the developed controller during velocity, angle of attack, and pitch-rate tracking, simulations for the full nonlinear model [11] are provided that include aerothermoelastic model uncertainties and nonlinear exogenous disturbances whose magnitude is based on airspeed fluctuations.

\section{HSV Model}

\section{A. Rigid Body and Elastic Dynamics}

To incorporate structural dynamics and aerothermoelastic effects in the HSV dynamic model, an assumed-modes model is considered for the longitudinal dynamics [ㅁ] as

$$
\begin{gathered}
\dot{V}=\frac{T \cos (\alpha)-D}{m}-g \sin (\theta-\alpha) \\
\dot{h}=V \sin (\theta-\alpha) \\
\dot{\alpha}=-\frac{L+T \sin (\alpha)}{m V}+Q+\frac{g}{V} \cos (\theta-\alpha) \\
\dot{\theta}=Q \\
\dot{Q}=\frac{M}{I_{y y}} \\
\ddot{\eta}_{i}=-2 \zeta_{i} \omega_{i} \dot{\eta}_{i}-\omega_{i}^{2} \eta_{i}+N_{i}, \quad i=1,2,3
\end{gathered}
$$

In Eqs. (1-6), $V(t) \in \mathbb{R}$ denotes the forward velocity; $h(t) \in \mathbb{R}$ denotes the altitude; $\alpha(t) \in \mathbb{R}$ denotes the angle of attack; $\theta(t) \in \mathbb{R}$ denotes the pitch angle; $Q(t) \in \mathbb{R}$ is pitch rate; and $\eta_{i}(t) \in \mathbb{R} \forall i=1$, 2, 3 denotes the $i$ th generalized structural mode displacement. Also in Eqs. (1-6), $m \in \mathbb{R}$ denotes the vehicle mass; $I_{y y} \in \mathbb{R}$ is the moment of inertia; $g \in \mathbb{R}$ is the acceleration due to gravity; $\zeta_{i}(t), \omega_{i}(t) \in \mathbb{R}$ are the damping factor and natural frequency of the $i$ th flexible mode, respectively; $T(x) \in \mathbb{R}$ denotes the thrust; $D(x) \in \mathbb{R}$ denotes the drag; $L(x) \in \mathbb{R}$ is the lift; $M(x) \in \mathbb{R}$ is the pitching moment about the body $y$ axis; and $N_{i}(x) \in \mathbb{R} \forall i=1,2,3$ denotes the generalized elastic forces, where $x(t) \in \mathbb{R}^{11}$ is composed of the five flight and six structural dynamic states as

$$
x=\left[\begin{array}{lllllllllll}
V & \alpha & Q & h & \theta & \eta_{1} & \dot{\eta}_{1} & \eta_{2} & \dot{\eta}_{2} & \eta_{3} & \dot{\eta}_{3}
\end{array}\right]^{T}
$$

The equations that define the aerodynamic and generalized moments and forces are highly coupled and are provided explicitly in previous work [1]. Specifically, the rigid-body and elastic modes are coupled in the sense that $T(x), D(x)$, and $L(x)$, are functions of $\eta_{i}(t)$ and that $N_{i}(x)$ is a function of the other states. As the temperature profile changes, the modulus of elasticity of the vehicle changes and the damping factors and natural frequencies of the flexible modes will change. The subsequent development exploits an implicit learning control structure, designed based on an LPV approximation of the dynamics in Eqs. (1-6), to yield exponential tracking despite the uncertainty due to the unknown aerothermoelastic effects and additional unmodeled dynamics.

\section{B. Temperature Profile Model}

Temperature variations impact the HSV flight dynamics through changes in the structural dynamics which affect the mode shapes and natural frequencies of the vehicle. The temperature model used assumes a free-free beam [1], which may not capture the actual aircraft dynamics properly. In reality, the internal structure will be made of a complex network of structural elements that will expand at different rates causing thermal stresses. Thermal stresses affect different modes in different manners, such that it raises the frequencies of some modes and lowers others (compared to a uniform degradation with Young's modulus only). Therefore, the current model only offers an approximate approach. The natural frequencies of a continuous beam are a function of the mass distribution of the beam and the stiffness. In turn, the stiffness is a function of Young's modulus $E$ and admissible mode functions. Hence, by modeling Young's modulus as a function of temperature, the effect of temperature on flight dynamics can be captured. Thermostructural dynamics are calculated under the material assumption that titanium is below the thermal protection system $[17,18]$. Young's modulus $E$ and the natural dynamic frequencies for the first three modes of a titanium free-free beam are depicted in Figs. 1 and 2 , respectively.

In Fig. 1, the moduli for the three modes are nearly identical. The temperature range shown corresponds to the temperature range that will be used in the simulation section. Frequencies in Fig. 2 correspond to a solid titanium beam, which will not correspond to the actual natural frequencies of the aircraft. The data shown in Fig. 1 and $\underline{2}$ are both from previous experimental work [19]. Using this data, different temperature gradients along the fuselage are introduced into the model and affect the structural properties of the HSV. The subsequent simulation uses linearly decreasing gradients from the nose to the tail section. It is expected that the nose will be the hottest part of the structure due to aerodynamic heating behind the bow shock wave. Thermostructural dynamics are calculated under the assumption that there are nine constant-temperature sections in the aircraft [20], as shown in Fig. 3. Since the aircraft is $100 \mathrm{ft}$ long, the length of each of the nine sections is approximately $11.1 \mathrm{ft}$.

An example of some of the mode frequencies are provided in Table 1, which shows the variation in the natural frequencies for five decreasing linear temperature profiles shown in Fig. 4. For all three natural modes, Table 1 shows that the natural frequency for the first temperature profile is almost $7 \%$ lower than that of the fifth temperature profile. The structural modes and frequencies are calculated using an assumed-modes method with finite element discretization, including vehicle mass distribution and inertia effects. The result of this method is the generalized mode shapes and mode frequencies for the HSV. Because the beam is nonuniform in temperature, the modulus of elasticity is also nonuniform, which

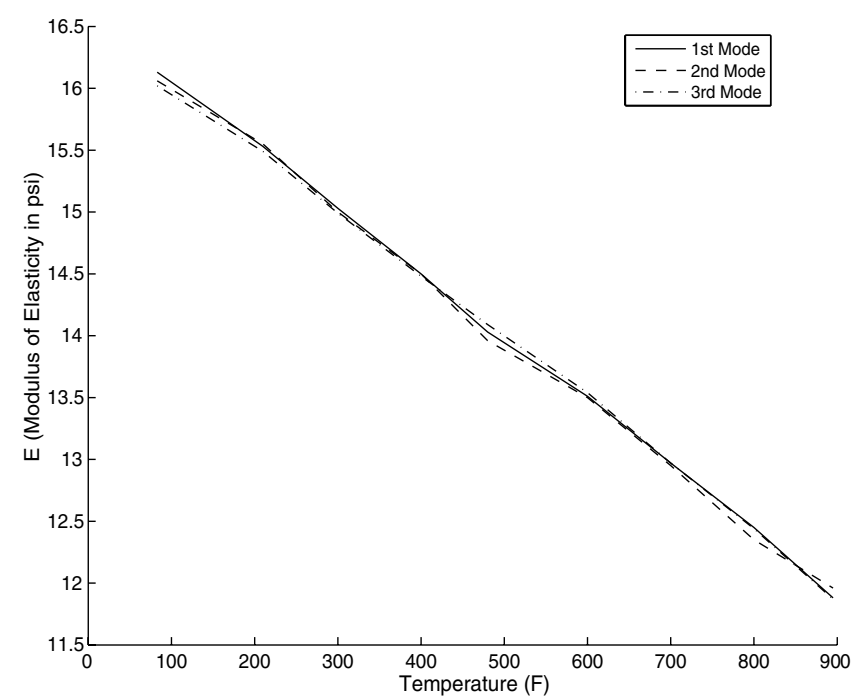

Fig. 1 Modulus of elasticity for the first three dynamic modes of vibration for a free-free beam of titanium. 

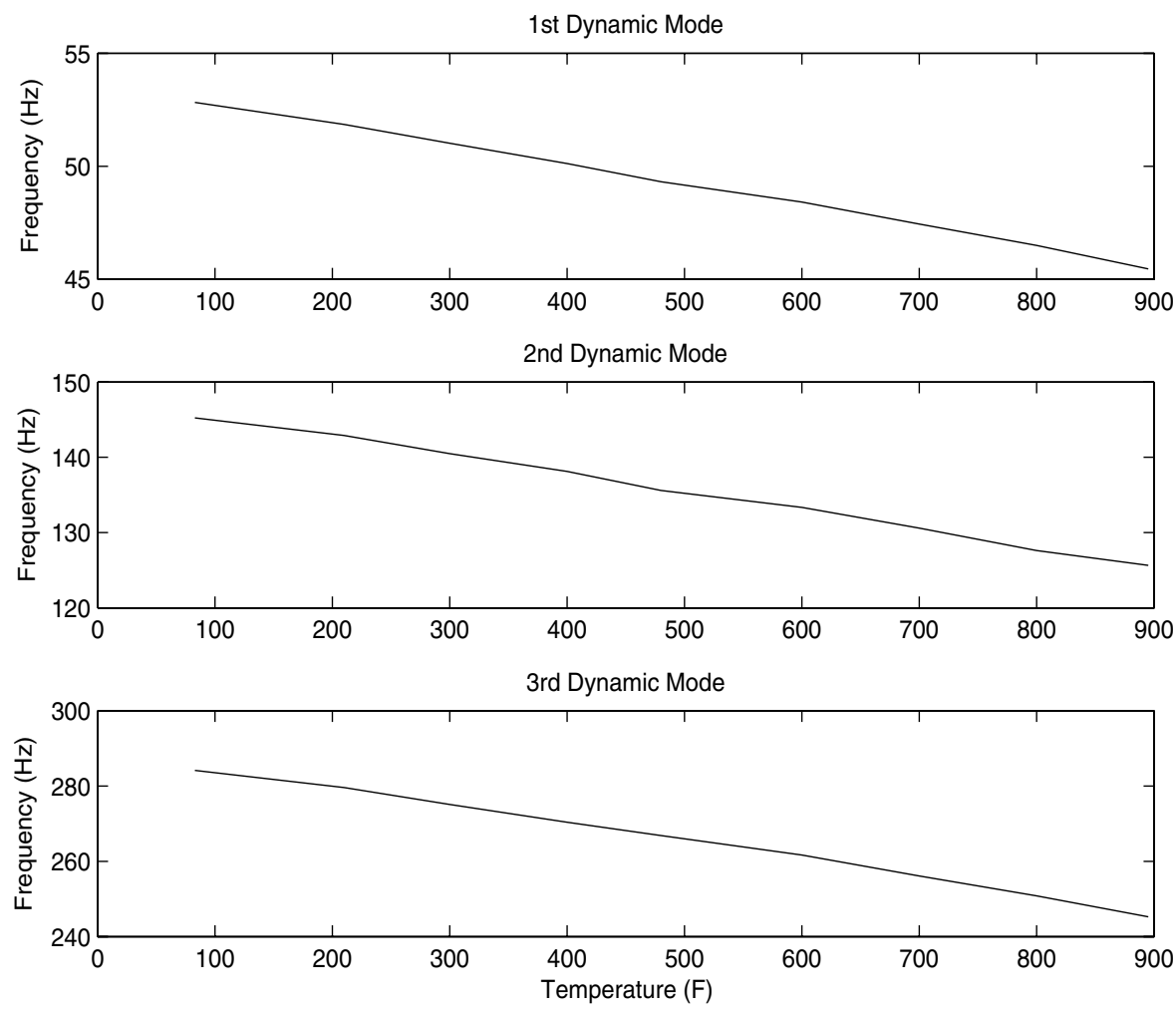

Fig. 2 Frequencies of vibration for the first three dynamic modes of a free-free titanium beam.

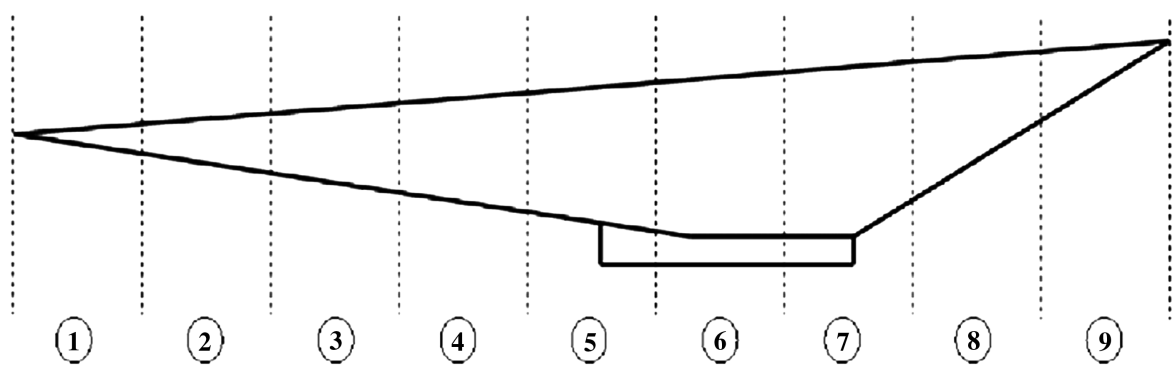

Fig. 3 Nine constant-temperature sections of the HSV used for temperature profile modeling.

produces asymmetric mode shapes. An example of the asymmetric mode shapes is shown in Fig. 5 and the asymmetry is due to variations in $E$ resulting from the fact that each of the nine fuselage sections (see Fig. 3) has a different temperature and hence different flexible dynamic properties.

The temperature profile in a HSV is a complex function of the state history, structural properties, thermal protection system, etc. In the

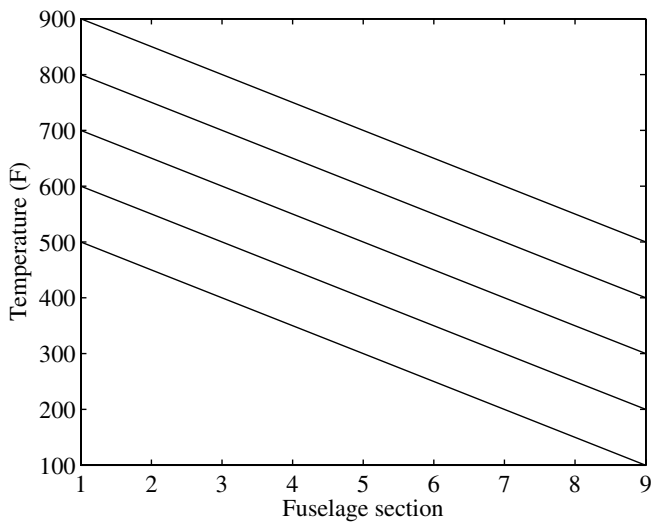

Fig. 4 Linear temperature profiles used to calculate values shown in Table 1 . subsequent simulation, the temperature profile is assumed to be a linear function that decreases from the nose to the tail of the aircraft. The linear profiles are then varied to span a preselected design space. Rather than attempting to model a physical temperature gradient for some vehicle design, the temperature profile in the simulation section is intended to provide an aggressive temperature-dependent profile to examine the robustness of the controller to such fluctuations.

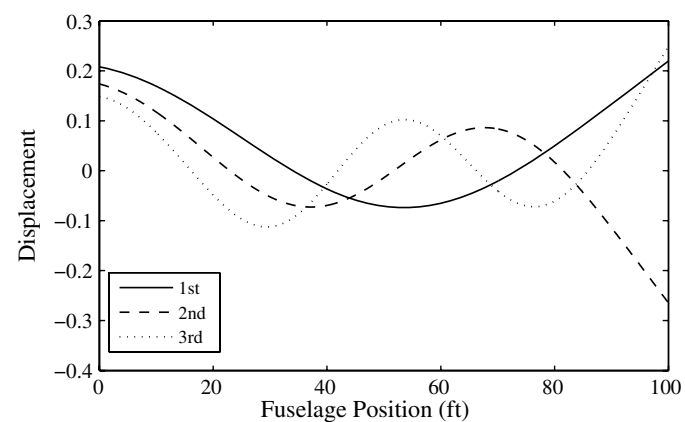

Fig. 5 Asymmetric mode shapes for the hypersonic vehicle. The percent difference was calculated based on the maximum minus the minimum structural frequencies divided by the minimum. 
Table 1 Natural frequencies for five linear temperature profiles (nose/tail) in ${ }^{\circ} \mathbf{F}$

\begin{tabular}{ccccccc}
\hline \hline & $900 / 500$ & $800 / 400$ & $700 / 300$ & $600 / 200$ & $500 / 100$ & $\%$ \\
Mode & $\mathrm{Hz}$ & $\mathrm{Hz}$ & $\mathrm{Hz}$ & \multicolumn{1}{c}{$\mathrm{Hz}$} & \multicolumn{1}{c}{$\mathrm{Hz}$} & difference \\
\hline 1 & 23.0 & 23.0 & 23.9 & 24.3 & 24.7 & $7.39 \%$ \\
2 & 49.9 & 50.9 & 51.8 & 52.6 & 53.5 & $7.21 \%$ \\
3 & 98.9 & 101.0 & 102.7 & 104.4 & 106.2 & $7.38 \%$ \\
\hline \hline
\end{tabular}

apercent difference is the difference between the maximum and minimum frequencies divided by the minimum frequency.

\section{Control Model}

The HSV dynamic model used for the subsequent control development is a combination of LPV state matrices and nonlinearity arising from unmodeled effects as $[\underline{4}, \underline{21}]$

$$
\begin{gathered}
\dot{x}=A(\rho(t)) x+B(\rho(t)) u+f(t) \\
y=C x
\end{gathered}
$$

In Eqs. (8) and (9), the state vector $x(t) \in \mathbb{R}^{11}$ is composed of the same five flight and six structural dynamic states described in Sec. II.A. Also in Eq. $(8), A(\rho(t)) \in \mathbb{R}^{11 \times 11}$ denotes a linearparameter-varying state matrix, $B(\rho(t)) \in \mathbb{R}^{11 \times p}$ denotes a columndeficient, linear-parameter-varying input matrix, $C \in \mathbb{R}^{p \times 11}$ denotes a known output matrix, $u(t) \in \mathbb{R}^{p}$ denotes a vector of $p$ control inputs, $\rho(t)$ represents the unknown time-dependent temperature profile of the aircraft, and $f(t) \in \mathbb{R}^{11}$ represents a time-dependent unknown nonlinear disturbance.

The matrices $A(\rho(t))$ and $B(\rho(t))$ have the standard linearparameter-varying form []] :

$$
\begin{aligned}
& A(\rho, t)=A_{0}+\sum_{i=1}^{s} w_{i}(\rho(t)) A_{i} \\
& B(\rho, t)=B_{0}+\sum_{i=1}^{s} v_{i}(\rho(t)) B_{i}
\end{aligned}
$$

where $A_{0} \in \mathbb{R}^{11 \times 11}$ and $B_{0} \in \mathbb{R}^{11 \times p}$ represent known nominal matrices with unknown variations $w_{i}(\rho(t)) A_{i}$ and $v_{i}(\rho(t)) B_{i}$ for $i=1,2, \ldots, s$, where $A_{i} \in \mathbb{R}^{11 \times 11}$ and $B_{i} \in \mathbb{R}^{11 \times p}$ are time-invariant matrices, and $w_{i}(\rho(t))$ and $v_{i}(\rho(t)) \in \mathbb{R}$ are parameter-dependent weighting terms. Knowledge of the nominal matrix $B_{0}$ will be exploited in the subsequent control design.

To facilitate the subsequent control design, a reference model is given as

$$
\begin{gathered}
\dot{x}_{m}=A_{m} x_{m}+B_{m} \delta \\
y_{m}=C x_{m}
\end{gathered}
$$

where $A_{m} \in \mathbb{R}^{11 \times 11}$ and $B_{m} \in \mathbb{R}^{11 \times p}$ denote the state and input matrices, respectively, where $A_{m}$ is Hurwitz; $\delta(t) \in \mathbb{R}^{p}$ is a vector of reference inputs; $y_{m}(t) \in \mathbb{R}^{p}$ are the reference outputs; and $C$ was defined in Eq. (9).

Assumption 1: The nonlinear disturbance $f(t)$ and its first two time derivatives are assumed to exist and be bounded by known constants.

Assumption 2: The dynamics in Eqs. ()ㅡㄹ and (9) are assumed to be controllable.

Assumption 3: The matrices $A(\rho(t))$ and $B(\rho(t))$ and their time derivatives satisfy the following inequalities:

$$
\begin{array}{cl}
\|A(\rho(t))\|_{i \infty} \leq \zeta_{A} \quad & \|B(\rho(t))\|_{i \infty} \leq \zeta_{B} \quad\|\dot{A}(\rho(t))\|_{i \infty} \leq \zeta_{A d} \\
& \|\dot{B}(\rho(t))\|_{i \infty} \leq \zeta_{B d}
\end{array}
$$

where $\zeta_{A}, \zeta_{B}, \zeta_{A d}$, and $\zeta_{B d} \in \mathbb{R}^{+}$are known bounding constants, and $\|\cdot\|_{i \infty}$ denotes the induced infinity norm of a matrix. As is typical in robust control methods, knowledge of the upper bounds in Eq. (14) are used to develop sufficient conditions on gains used in the subsequent control design.

\section{Control Development}

\section{A. Control Objective}

The control objective is to ensure that the output $y(t)$ tracks the time-varying output generated from the reference model in Eqs. (12) and (13). To quantify the control objective, an output tracking error, denoted by $e(t) \in \mathbb{R}^{p}$, is defined as

$$
e \triangleq y-y_{m}=C\left(x-x_{m}\right)
$$

To facilitate the subsequent analysis, a filtered tracking error denoted by $r(t) \in \mathbb{R}^{p}$, is defined as

$$
r \triangleq \dot{e}+\gamma e
$$

where $\gamma \in \mathbb{R}$ is a positive, constant control gain, and is selected to place a relative weight on the error state verses its derivative. To facilitate the subsequent robust control development, the state vector $x(t)$ is expressed as

$$
x(t)=\underline{x}(t)+x_{u}(t)
$$

where $\underline{x}(t) \in \mathbb{R}^{11}$ contains the $p$ output states, and $x_{u}(t) \in \mathbb{R}^{11}$ contains the remaining $11-p$ states. Likewise, the reference states $x_{m}(t)$ can also be separated as in Eq. (17).

Assumption 4: The states contained in $x_{u}(t)$ in Eq. (17) and the corresponding time derivatives can be further separated as

$$
x_{u}(t)=x_{\rho u}(t)+x_{\zeta u}(t) \quad \dot{x}_{u}(t)=\dot{x}_{\rho u}(t)+\dot{x}_{\zeta u}(t)
$$

where $x_{\rho u}(t), \dot{x}_{\rho u}(t), x_{\zeta u}(t)$, and $\dot{x}_{\zeta u}(t) \in \mathbb{R}^{11}$ are upper-bounded as

$$
\begin{aligned}
& \left\|x_{\rho u}(t)\right\| \leq c_{1}\|z\| \quad\left\|x_{\zeta u}(t)\right\| \leq \zeta_{x u} \quad\left\|\dot{x}_{\rho u}(t)\right\| \leq c_{2}\|z\| \\
& \left\|\dot{x}_{\zeta u}(t)\right\| \leq \zeta_{\dot{x} u}
\end{aligned}
$$

where $z(t) \in \mathbb{R}^{2 p}$ is defined as

$$
z \triangleq\left[\begin{array}{ll}
e^{T} & r^{T}
\end{array}\right]^{T}
$$

and $c_{1}, c_{2}, \zeta_{x u}$, and $\zeta_{\dot{x} u} \in \mathbb{R}$ are known nonnegative bounding constants. The terms in Eqs. (17) and (19) are used to develop sufficient gain conditions for the subsequent robust control design.

\section{B. Open-Loop Error System}

The open-loop tracking error dynamics can be developed by taking the time derivative of Eq. (16) and using the expressions in

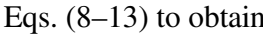

$$
\begin{aligned}
\dot{r}= & \ddot{e}+\gamma \dot{e}=C\left(\ddot{x}-\ddot{x}_{m}\right)+\gamma \dot{e}=C(\dot{A} x+A \dot{x}+\dot{B} u+B \dot{u}+\dot{f}(t) \\
& \left.-A_{m} \dot{x}_{m}-B_{m} \dot{\delta}\right)+\gamma \dot{e}=\tilde{N}+N_{d}+C \dot{B} u+C B \dot{u}-e
\end{aligned}
$$

The auxiliary functions $\tilde{N}\left(x, \dot{x}, e, x_{m}, \dot{x}_{m}, t\right) \in \mathbb{R}^{p} \quad$ and $N_{d}\left(x_{m}, \dot{x}_{m}, \delta, \dot{\delta}, t\right) \in \mathbb{R}^{p}$ in Eq. (21) are defined as

$$
\tilde{N} \triangleq C A\left(\dot{x}-\dot{x}_{m}\right)+C \dot{A}\left(\underline{x}-\underline{x}_{m}\right)+C A \dot{x}_{\rho u}+C \dot{A} x_{\rho u}+\gamma \dot{e}+e
$$

and

$$
\begin{aligned}
N_{d} & \triangleq C \dot{f}(t)+C A \dot{x}_{\zeta u}+C \dot{A} x_{\zeta u}+C A \dot{x}_{m}+C \dot{A} \underline{x}_{m} \\
& -C A_{m} \dot{x}_{m}-C B_{m} \dot{\delta}
\end{aligned}
$$

Motivation for the selective grouping of the terms in Eqs. (22) and (23) is derived from the fact that the following inequalities can be developed $[22,23]$ : 


$$
\|\tilde{N}\| \leq \rho_{0}\|z\| \quad\left\|N_{d}\right\| \leq \zeta_{N_{d}}
$$

where $\rho_{0}, \zeta_{N_{d}} \in \mathbb{R}^{+}$are known bounding constants.

\section{Closed-Loop Error System}

Based on the expression in Eq. (21) and the subsequent stability analysis, the control input is designed as

$$
u=-k_{\Gamma}\left(C B_{0}\right)^{-1}\left[\left(k_{s}+I_{p \times p}\right) e(t)-\left(k_{s}+I_{p \times p}\right) e(0)+v(t)\right]
$$

where $v(t) \in \mathbb{R}^{p}$ is an implicit learning law with an update rule given by

$$
\dot{v}(t)=k_{u}\|u(t)\| \operatorname{sgn}(r(t))+\left(k_{s}+I_{p \times p}\right) \gamma e(t)+k_{\gamma} \operatorname{sgn}(r(t))
$$

and $k_{\Gamma}, k_{u}, k_{s}$, and $k_{\gamma} \in \mathbb{R}^{p \times p}$ denote positive-definite diagonal constant-control-gain matrices; $B_{0} \in \mathbb{R}^{11 \times p}$ is introduced in Eq. (11), $\operatorname{sgn}(\cdot)$ denotes the standard signum function, in which the function is applied to each element of the vector argument; and $I_{p \times p}$ denotes a $p \times p$ identity matrix.

After substituting the time derivative of Eq. (25) into Eq. (21), the error dynamics can be expressed as

$$
\begin{aligned}
\dot{r}= & \tilde{N}+N_{d}-\tilde{\Omega} k_{u}\|u(t)\| \operatorname{sgn}(r(t))+C \dot{B} u-\tilde{\Omega}\left(k_{s}+I_{p \times p}\right) r(t) \\
& -\tilde{\Omega} k_{\gamma} \operatorname{sgn}(r(t))-e
\end{aligned}
$$

where the auxiliary matrix $\tilde{\Omega}(\rho(t)) \in \mathbb{R}^{p \times p}$ is defined as

$$
\tilde{\Omega} \triangleq C B k_{\Gamma}\left(C B_{0}\right)^{-1}
$$

where $\tilde{\Omega}(\rho(t))$ can be separated into diagonal [i.e., $\Lambda(\rho(t)) \in \mathbb{R}^{p \times p}$ ] and offdiagonal [i.e., $\Delta(\rho(t)) \in \mathbb{R}^{p \times p}$ ] components as

$$
\tilde{\Omega}=\Lambda+\Delta
$$

Differential equations such as Eqs. (25) and (26) have discontinuous right-hand sides. Let $f(y, t) \in \overline{\mathbb{R}^{2} p}$ denote the righthand side of Eqs. (25) and (26). Since the subsequent analysis requires that a solution exist for $\dot{y}=f(y, t)$, it is important to show the existence of the generalized solution. The existence of Fillipov's generalized solution [24] can be established for Eqs. (25) and (26).

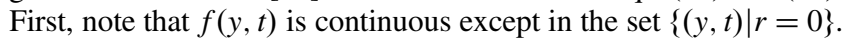
Let $F(y, t)$ be a compact, convex, upper semicontinuous set-valued map that embeds the differential equation $\dot{y}=f(y, t)$ into the differential inclusions $\dot{y} \in F(y, t)$. An absolute continuous solution exists to $\dot{y} \in F(y, t)$ that is a generalized solution to $\dot{y}=f(y, t)$. A common choice [24] for $F(y, t)$ that satisfies the above conditions is the closed convex hull of $f(y, t)$. A proof that this choice for $F(y, t)$ is upper semicontinuous is given in [25].

Assumption 5: The subsequent development is based on the assumption that the uncertain matrix $\tilde{\Omega}(\rho(t))$ is diagonally dominant in the sense that

$$
\lambda_{\min }(\Lambda)-\|\Delta\|_{i \infty}>\varepsilon
$$

where $\varepsilon \in \mathbb{R}^{+}$is a known constant. While this assumption cannot be validated for a generic HSV, the condition can be checked (within some certainty tolerances) for a given aircraft. Essentially, this condition indicates that the nominal value $B_{0}$ introduced in Eq. (11) and used in the controller in Eq. (25) must remain within some bounded region of $B$. In practice, bands on the variation of $B$ should be known, for a particular aircraft under a set of operating conditions, and this band could be used to check the sufficient conditions given in Eq. (30).

Motivation for the structure of the controller in Eqs. (25) and (26) comes from the desire to develop a closed-loop error system to facilitate the subsequent Lyapunov-based stability analysis. In particular, since the control input is premultiplied by the uncertain matrix $C B$ in Eq. (21), the term $\left(C B_{0}\right)^{-1}$ is motivated to generate the relationship in Eq. (28) so that if the diagonal dominance assumption (Assumption 5) is satisfied, then the control can provide feedback to compensate for the disturbance terms. The bracketed terms in Eq. (25) include the state feedback, an initial condition term, and the implicit learning term. The implicit learning term $v(t)$ is the generalized solution to Eq. (26). The structure of the update law in Eq. (26) is motivated by the need to reject the exogenous disturbance terms. Specifically, the update law is motivated by a sliding mode control strategy that can be used to eliminate additive bounded disturbances. Unlike sliding mode control (which is a discontinuous control method requiring infinite actuator bandwidth), the current continuous control approach includes the integral of the $\operatorname{sgn}()$ function. This implicit learning law is the key element that allows the controller to obtain an exponential stability result despite the additive nonvanishing exogenous disturbance. Other results in literature also have used the implicit learning structure [26-29].

\section{Stability Analysis}

Theorem 1: The controller given in Eqs. (25) and (26) ensures exponential tracking in the sense that

$$
\|e(t)\| \leq\|z(0)\| \exp \left(-\frac{\lambda_{1}}{2} t\right) \quad \forall t \in[0, \infty)
$$

where $\lambda_{1} \in \mathbb{R}^{+}$, provided that the control gains $k_{u}, k_{s}$, and $k_{\gamma}$ introduced in Eq. (25) are selected according to the sufficient conditions $\stackrel{* *}{*}$

$$
\lambda_{\text {min }}\left(k_{u}\right) \geq \frac{\bar{\zeta}_{B d}}{\varepsilon} \quad \lambda_{\text {min }}\left(k_{s}\right)>\frac{\rho_{0}^{2}}{4 \varepsilon \min \{\gamma, \varepsilon\}} \quad \lambda_{\text {min }}\left(k_{\gamma}\right)>\frac{\zeta_{N_{d}}}{\varepsilon}
$$

where $\rho_{0}$ and $\zeta_{N_{d}}$ are introduced in Eq. (24), $\varepsilon$ is introduced in Eq. (30), $\bar{\zeta}_{B d} \in \mathbb{R}^{+}$is a known positive constant, and $\lambda_{\min }(\cdot)$ denotes the minimum eigenvalue of the argument.

Proof: Let $V_{L}(z, t): \mathbb{R}^{2 p} \times[0, \infty) \rightarrow \mathbb{R}$ be a continuously differentiable, positive-definite function defined as

$$
V_{L}(z, t) \triangleq \frac{1}{2} e^{T} e+\frac{1}{2} r^{T} r
$$

where $e(t)$ and $r(t)$ are defined in Eqs. (15) and (16), respectively. After taking the time derivative of Eq. (33) and using Eqs. (16), (27), and (29), $\dot{V}_{L}(z, t)$ can be expressed as

$$
\begin{aligned}
& \dot{V}_{L}(z, t)=-\gamma e^{T} e+r^{T} \tilde{N}+r^{T} C \dot{B} u-r^{T} \Lambda\left(k_{s}+I_{p \times p}\right) r \\
& \quad-r^{T} \Delta\left(k_{s}+I_{p \times p}\right) r-r^{T} \Lambda\|u\| k_{u} \operatorname{sgn}(r)-r^{T} \Delta\|u\| k_{u} \operatorname{sgn}(r) \\
& \quad-r^{T} \Lambda k_{\gamma} \operatorname{sgn}(r)-r^{T} \Delta k_{\gamma} \operatorname{sgn}(r)+r^{T} N_{d}
\end{aligned}
$$

By using the bounding arguments in Eq. (24) and Assumptions 3 and 5, the upper bound of the expression in Eq. (34) can be explicitly determined. Specifically, based on Eq. (14) of Assumption 3, the term $r^{T} C \dot{B} u$ in Eq. (34) can be upper-bounded as

$$
r^{T} C \dot{B} u \leq \bar{\zeta}_{B d}\|r\|\|u\|
$$

After using inequality (30) of Assumption 5, the following inequalities can be developed:

$$
\begin{aligned}
- & r^{T} \Lambda\left(k_{s}+I_{p \times p}\right) r-r^{T} \Delta\left(k_{s}+I_{p \times p}\right) r \leq-\varepsilon\left(\lambda_{\min }\left(k_{s}\right)+1\right)\|r\|^{2} \\
& -r^{T} \Lambda\|u(t)\| k_{u} \operatorname{sgn}(r)-r^{T} \Delta\|u(t)\| k_{u} \operatorname{sgn}(r) \\
& \leq-\varepsilon \lambda_{\min }\left(k_{u}\right)|r|\|u\|-r^{T} \Lambda k_{\gamma} \operatorname{sgn}(r)-r^{T} \Delta k_{\gamma} \operatorname{sgn}(r) \\
& \leq-\varepsilon \lambda_{\min }\left(k_{\gamma}\right)|r|
\end{aligned}
$$

**The bounding constants are conservative upper bounds on the maximum expected values. The Lyapunov analysis indicates that the gains in Eq. (32) need to be selected sufficiently large based on the bounds. Therefore, if the constants are chosen to be conservative, then the sufficient gain conditions will be larger. Values for these gains could be determined through a physical understanding of the system (within some conservative percentage of uncertainty) and/or through numerical simulations. 
After using the inequalities in Eqs. (35) and (36), the expression in Eq. (34) can be upper-bounded as

$$
\begin{gathered}
\dot{V}_{L}(z, t) \leq-\gamma\|e\|^{2}+r^{T} \tilde{N}+\bar{\zeta}_{B d}\|r\|\|u\|-\varepsilon\left(\lambda_{\min }\left(k_{s}\right)+1\right)\|r\|^{2} \\
\quad-\varepsilon \lambda_{\text {min }}\left(k_{u}\right)\|r\|\|u\|-\varepsilon \lambda_{\text {min }}\left(k_{\gamma}\right)\|r\|+r^{T} N_{d}
\end{gathered}
$$

where the fact that $|r| \geq\|r\| \forall r \in \mathbb{R}^{p}$ was used. After using the inequalities in Eq. (24) and rearranging the resulting expression, the upper bound for $\dot{V}_{L}(z, t)$ can be expressed as

$$
\begin{gathered}
\dot{V}_{L}(z, t) \leq-\gamma\|e\|^{2}-\varepsilon\|r\|^{2}-\varepsilon \lambda_{\text {min }}\left(k_{s}\right)\|r\|^{2}+\rho_{0}\|r\|\|z\| \\
-\left[\varepsilon \lambda_{\text {min }}\left(k_{u}\right)-\zeta_{B d}\right]\|r\|\|u\|-\left[\varepsilon \lambda_{\text {min }}\left(k_{\gamma}\right)-\zeta_{N_{d}}\right]\|r\|
\end{gathered}
$$

If $k_{u}$ and $k_{\gamma}$ satisfy the sufficient gain conditions in Eq. (32), the bracketed terms in Eq. (38) are positive, and $\dot{V}_{L}(z, t)$ can be upperbounded using the squares of the components of $z(t)$ as

$$
\dot{V}_{L}(z, t) \leq-\gamma\|e\|^{2}-\varepsilon\|r\|^{2}-\left[\varepsilon \lambda_{\text {min }}\left(k_{s}\right)\|r\|^{2}-\rho_{0}\|r\|\|z\|\right]
$$

By completing the squares, the upper bound in Eq. (39) can be expressed in a more convenient form. To this end, the term

$$
\frac{\rho_{0}^{2}\|z\|^{2}}{4 \varepsilon \lambda_{\min }\left(k_{s}\right)}
$$

is added and subtracted to the right-hand side of Eq. (39), yielding

$$
\begin{aligned}
& \dot{V}_{L}(z, t) \leq-\gamma\|e\|^{2}-\varepsilon\|r\|^{2}-\varepsilon \lambda_{\min }\left(k_{s}\right)\left[\|r\|-\frac{\rho_{0}\|z\|}{2 \varepsilon \lambda_{\min }\left(k_{s}\right)}\right]^{2} \\
& \quad+\frac{\rho_{0}^{2}\|z\|^{2}}{4 \varepsilon \lambda_{\min }\left(k_{s}\right)}
\end{aligned}
$$

Since the square of the bracketed term in Eq. (40) is always positive, the upper bound can be expressed as

$$
\dot{V}_{L}(z, t) \leq-z^{T} \operatorname{diag}\left\{\gamma I_{p \times p}, \varepsilon I_{p \times p}\right\} z+\frac{\rho_{0}^{2}\|z\|^{2}}{4 \varepsilon \lambda_{\min }\left(k_{s}\right)}
$$

where $z(t)$ is defined in Eq. (20). Hence, Eq. (41) can be used to rewrite the upper bound of $\dot{V}_{L} \overline{(z, t)}$ as

$$
\dot{V}_{L}(z, t) \leq-\left(\min \{\gamma, \varepsilon\}-\frac{\rho_{0}^{2}}{4 \varepsilon \lambda_{\min }\left(k_{s}\right)}\right)\|z\|^{2}
$$

where the fact that $z^{T} \operatorname{diag}\left\{\gamma I_{p \times p}, \varepsilon I_{p \times p}\right\} z \geq \min \{\gamma, \varepsilon\}\|z\|^{2}$ was used.

Provided the gain condition in Eq. (32) is satisfied, Eqs. (33) and (42) can be used to show that $V_{L}(t) \in L_{\infty}$; hence, $e \overline{(t)}$ and $r(\bar{t}) \in L_{\infty}$. Given that $e(t)$ and $r(t) \in L_{\infty}$, standard linear analysis methods can be used to prove that $\dot{e}(t) \in L_{\infty}$ from Eq. (16). Since $e(t)$ and $\dot{e}(t) \in L_{\infty}$, the assumption that the reference model outputs $y_{m}(t)$ and $\dot{y}_{m}(t) \in L_{\infty}$ can be used along with Eq. (15) to prove that $y(t)$ and $\dot{y}(t) \in L_{\infty}$. Given that $y(t), \dot{y}(t), e(t)$, and $r(t) \in L_{\infty}$, the vector $\underline{x}(t) \in L_{\infty}$, the time derivative $\dot{x}(t) \in L_{\infty}$, and Eqs. (17-19) can be used to show that $x(t)$ and $\dot{x}(t) \in L_{\infty}$. Given that $x \overline{(t)}$ and $\dot{x}(t) \in L_{\infty}$, Assumptions 1, 2, and 3 can be used along with Eq. ( $\left.\underline{8}\right)$ to show that $u(t) \in L_{\infty}$

The definition for $V_{L}(z, t)$ in Eq. (33) can be used along with inequality (42) to show that $V_{L}(z, t)$ can be upper-bounded as

$$
\dot{V}_{L}(z, t) \leq-\lambda_{1} V_{L}(z, t)
$$

provided the sufficient condition in Eq. (32) is satisfied. The differential inequality in Eq. (43) can be solved as

$$
V_{L}(z, t) \leq V_{L}(z(0), 0) \exp \left(-\lambda_{1} t\right)
$$

Hence, Eqs. (20), (33), and (44) can be used to conclude that

$$
\|e(t)\| \leq\|z(0)\| \exp \left(-\frac{\lambda_{1}}{2} t\right) \quad \forall t \in[0, \infty)
$$

\section{Simulation}

The controller in Eqs. (25) and (26) and the associated stability analysis is based on the simplified linear-parameter-varying with additive disturbances dynamics given in Eqs. (7) and (8). To illustrate the performance of the controller and practicality of the assumptions, a numerical simulation was performed on the full nonlinear longitudinal equations of motion [1] given in Eqs. (1-6). The control inputs were selected as $u=\left[\begin{array}{lll}\delta_{e}(t) & \delta_{c}(t) & \phi_{f}(t)\end{array}\right]^{T}$, as in previous research [15], where $\delta_{e}(t)$ and $\delta_{c}(t)$ denote the elevator and canard deflection angles, respectively, $\phi_{f}(t)$ is the fuel equivalence ratio.
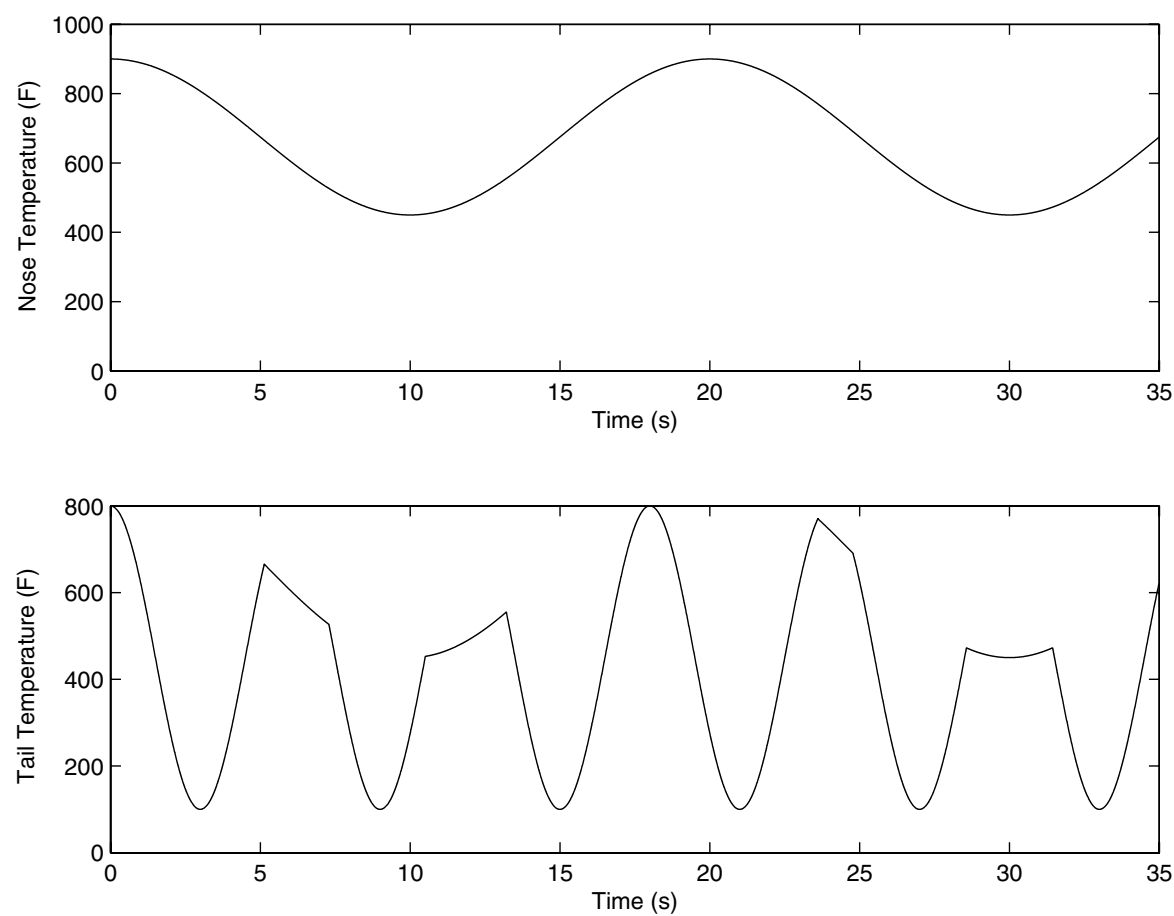

Fig. 6 Temperature variation for the forebody and aftbody of the hypersonic vehicle as a function of time. 

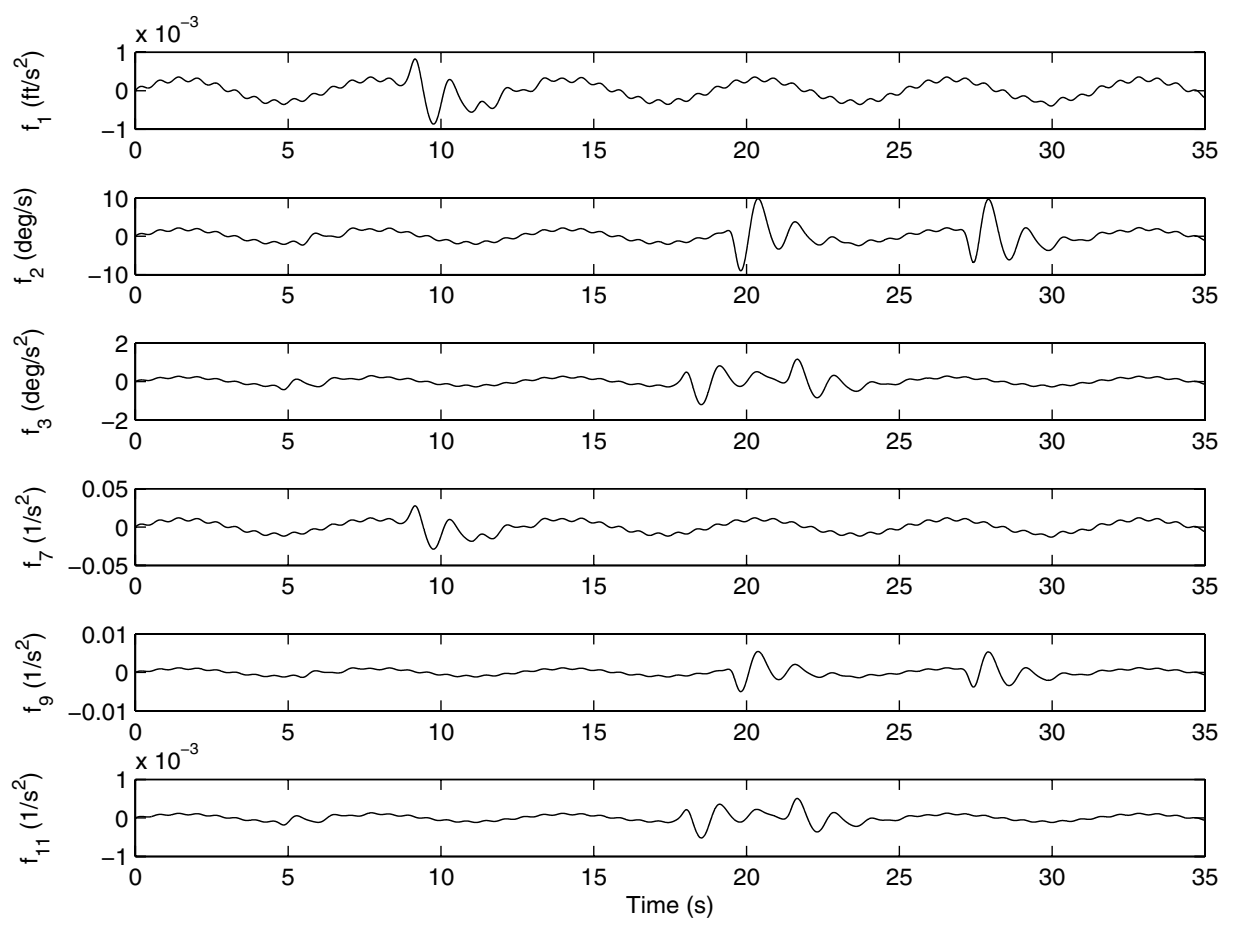

Fig. 7 In this figure, $f_{i}$ denotes the $i$ th element in the disturbance vector $f$. Disturbances from top to bottom: velocity $f_{\dot{V}}$, angle of attack $f_{\dot{\alpha}}$, pitch rate $f_{\dot{Q}}$, the first elastic structural mode $\ddot{\eta}_{1}$, the second elastic structural mode $\ddot{\eta}_{2}$, and the third elastic structural mode $\ddot{\eta}_{3}$, as described in Eq. (47).

The diffuser area ratio is left at its operational trim condition without loss of generality $\left(A_{d}(t)=1\right)$. The reference outputs were selected as maneuver oriented outputs of velocity, angle of attack, and pitch rate as $y=\left[\begin{array}{lll}V(t) & \alpha(t) & Q(t)\end{array}\right]^{T}$, where the output and state variables are introduced in Eqs. (1- $\underline{5})$. In addition, the proposed controller could be used to control other output states such as altitude, provided that the condition in Eq. (30) is satisfied.

The HSV parameters used in the simulation are $m=75,000 \mathrm{lb}$, $I_{y y}=86,723 \mathrm{lb} \cdot \mathrm{ft}^{2}$, and $g=32.174 \mathrm{ft} / \mathrm{s}^{2}$ as defined in Eqs. (1-6). The simulation was executed for $35 \mathrm{~s}$ to sufficiently cycle through the different temperature profiles. Other vehicle parameters in the simulation are functions of the temperature profile. Linear temperature profiles between the fore (i.e., $\left.T_{f b} \in[450,900]\right)$ and aft (i.e., $\left.T_{a b} \in[100,800]\right)$ were used to generate elastic mode shapes and frequencies by varying the linear gradients as

$$
\begin{aligned}
T_{f b}(t)=675+225 \cos \left(\frac{\pi}{10} t\right) \\
T_{a b}(t)= \begin{cases}450+350 \cos \left(\frac{\pi}{3} t\right) & \text { if } T_{f b}(t)>T_{a b}(t) \\
T_{f b}(t) & \text { otherwise }\end{cases}
\end{aligned}
$$

Figure 6 shows the temperature variation as a function of time. The irregularities seen in the aftbody temperatures occur because the temperature profiles were adjusted to ensure the tail of the aircraft
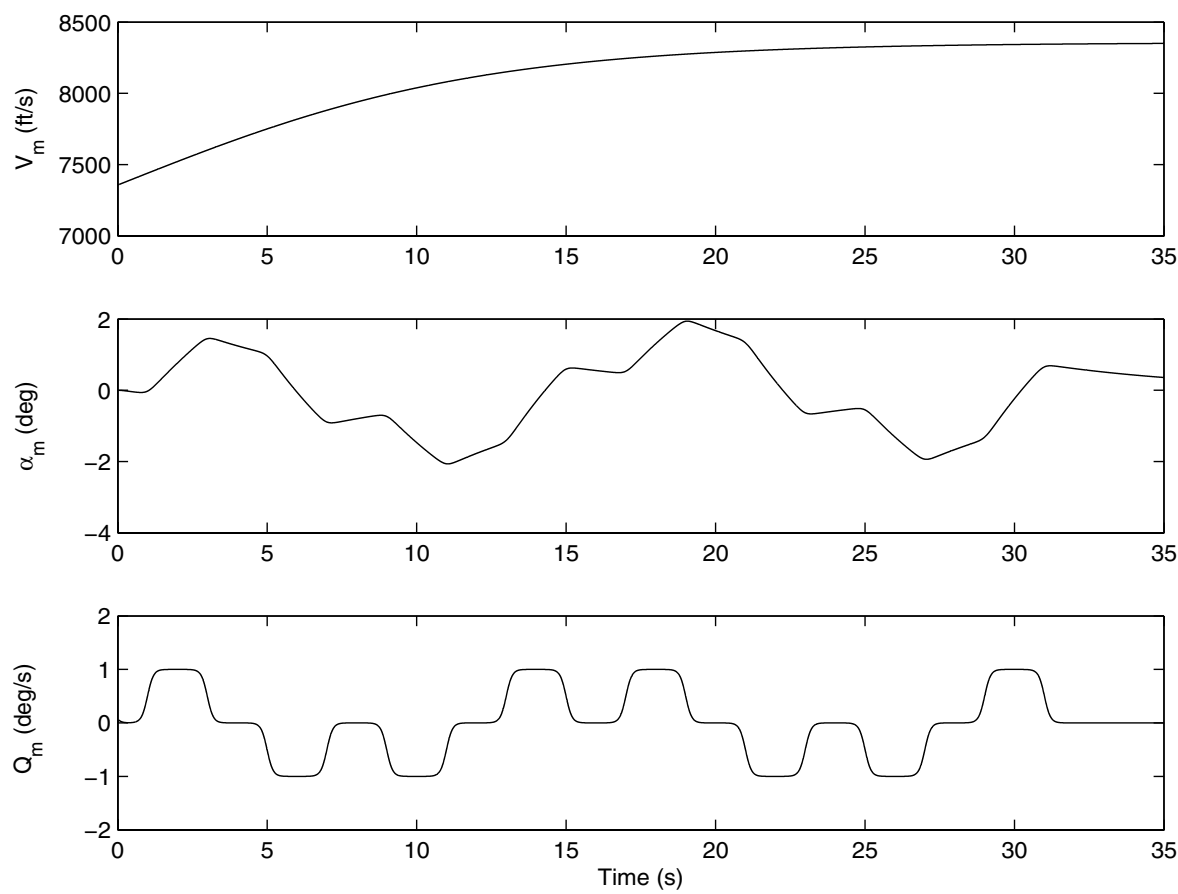

Fig. 8 Reference model outputs $y_{m}$, which are the desired trajectories for top: velocity $V_{m}(t)$, middle: angle of attack $\alpha_{m}(t)$, and bottom: pitch rate $Q_{m}(t)$. 

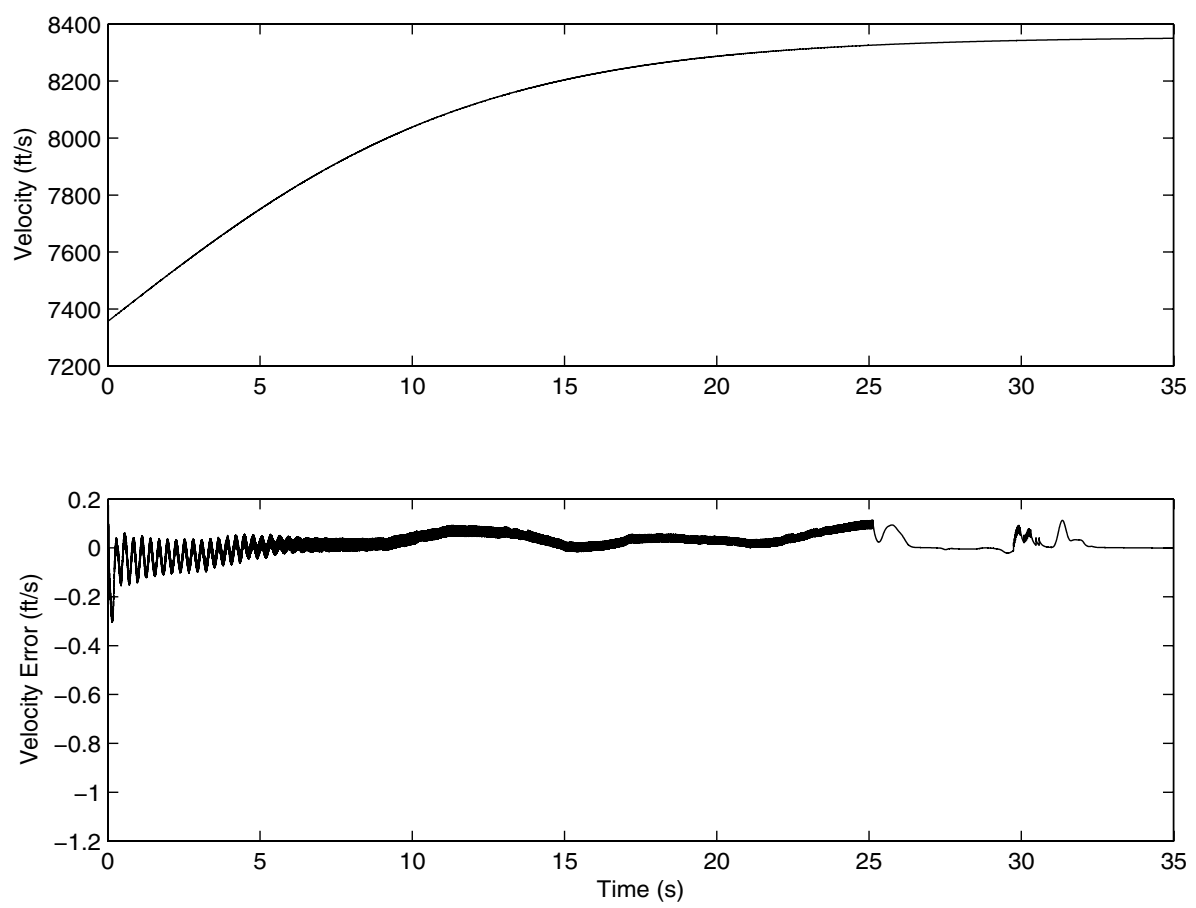

Fig. 9 Top: velocity $V(t)$ and bottom: velocity tracking error $e_{V}(t)$.

was equal or cooler than the nose of the aircraft according to bow shock wave thermodynamics. While the shock wave thermodynamics motivated the need to only consider the case when the tail of the aircraft was equal or cooler than the nose of the aircraft, the shape of the temperature profile is not physically motivated. Specifically, the frequencies of oscillation in Eq. (46) were selected to aggressively span the available temperature ranges. These temperature profiles are not motivated by physical temperature gradients, but motivated by the desire to generate an aggressive temperature disturbance to illustrate the controller robustness to the temperature gradients. The simulation assumes the damping coefficient remains constant for the structural modes $\left(\zeta_{i}=0.02\right)$.

In addition to thermoelasticity, a bounded nonlinear disturbance was added to the dynamics as

$$
f=\left[\begin{array}{lllllllllll}
f_{\dot{V}} & f_{\dot{\alpha}} & f_{\dot{Q}} & 0 & 0 & 0 & f_{\ddot{\eta} 1} & 0 & f_{\ddot{\eta} 2} & 0 & f_{\ddot{\eta} 3}
\end{array}\right]^{T}
$$

where $f_{\dot{V}}(t) \in \mathbb{R}$ denotes a longitudinal acceleration disturbance, $f_{\dot{\alpha}}(t) \in \mathbb{R}$ denotes a angle-of-attack rate-of-change disturbance, $f_{\dot{Q}}(t) \in \mathbb{R}$ denotes an angular acceleration disturbance, and $f_{\ddot{\eta} 1}(t)$, $f_{\ddot{\eta} 2}(t), f_{\ddot{\eta} 3}(t), \in \mathbb{R}$ denote structural mode acceleration disturbances. The disturbances in Eq. (47) were generated as an arbitrary exogenous input (i.e., unmodeled nonvanishing disturbance that does not satisfy the linear in the parameters assumption) as depicted in Fig. 7. However, the magnitudes of the disturbances were motivate $\bar{d}$ by the scenario of a $300 \mathrm{ft} / \mathrm{s}$ change in airspeed. The disturbances are not designed to mimic the exact effects of a wind gust, but to demonstrate the proposed controller's robustness with
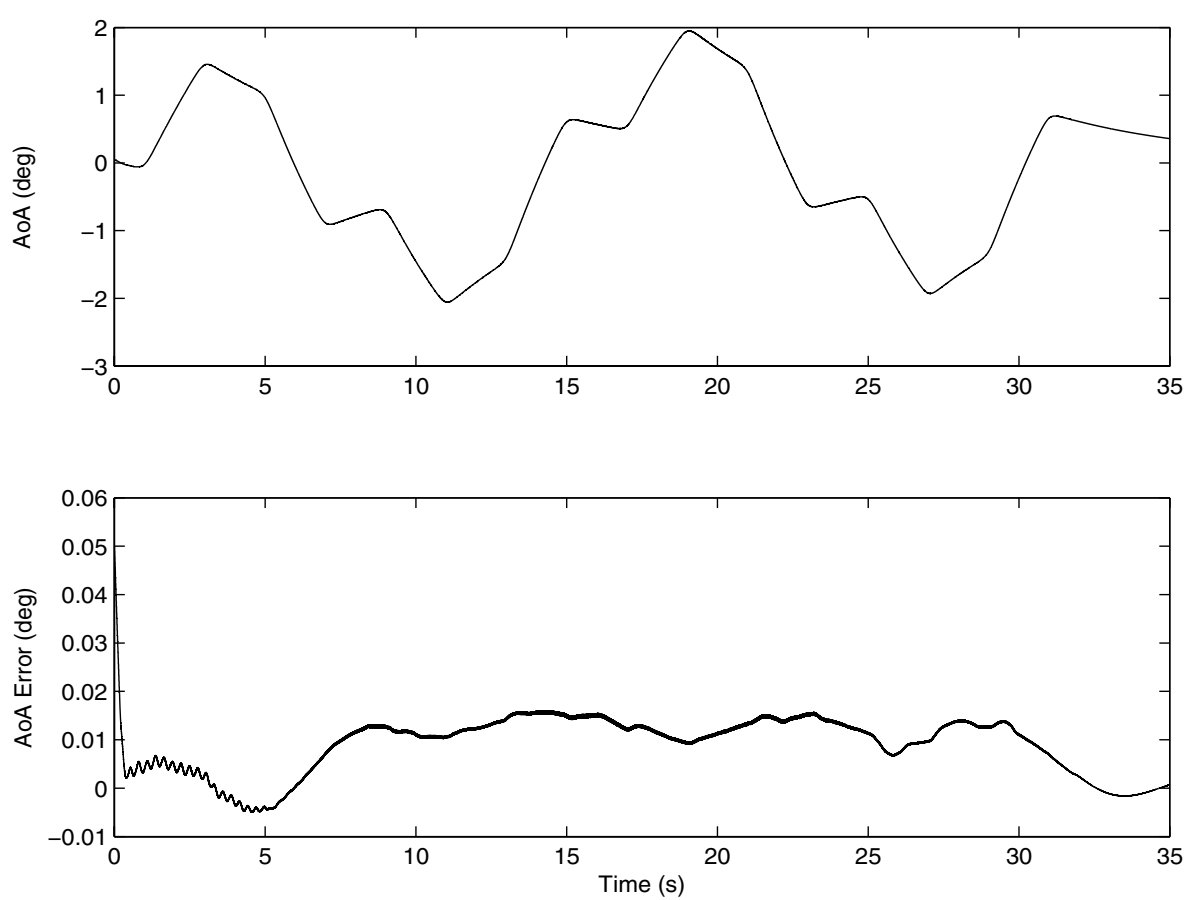

Fig. 10 Top: angle of attack $\alpha(t)$ and bottom: angle-of-attack tracking error $e_{\alpha}(t)$. 

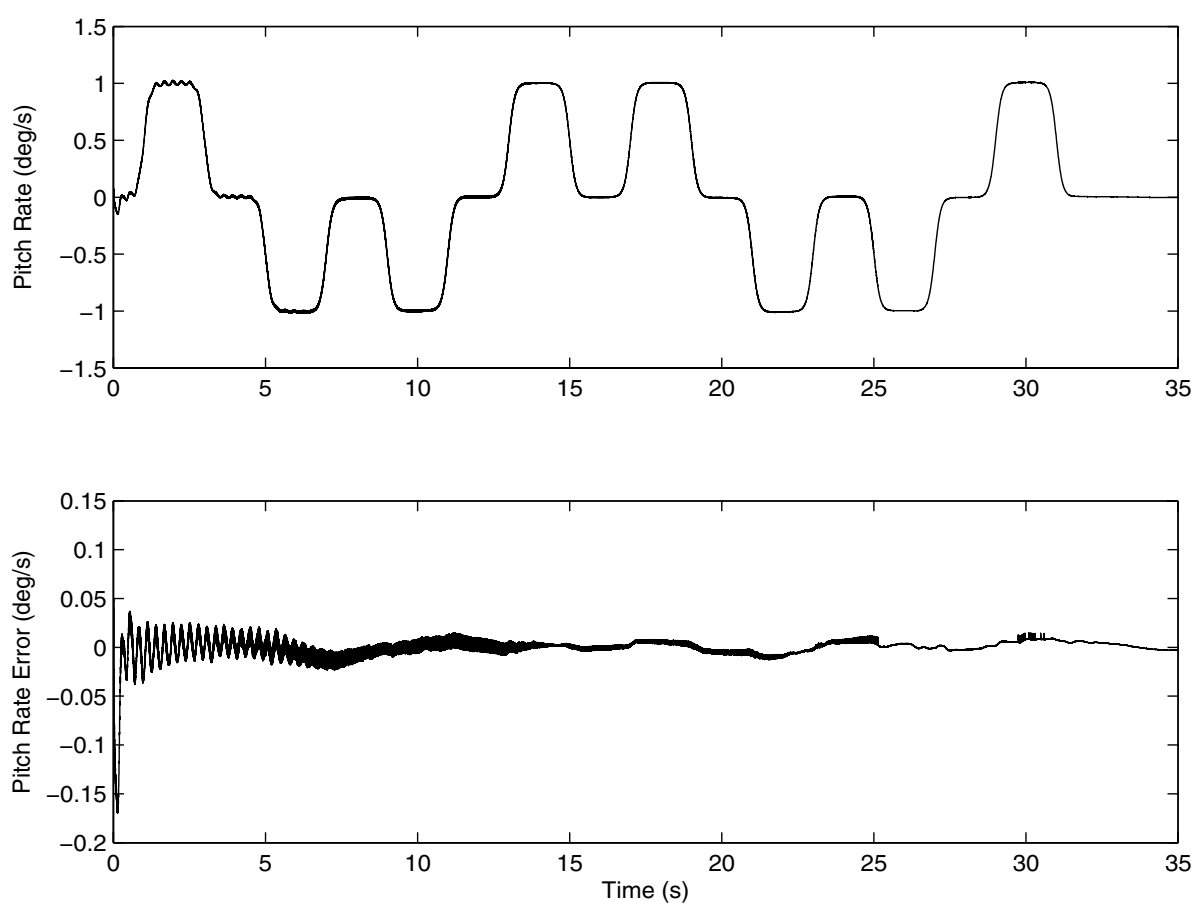

Fig. 11 Top: pitch rate $Q(t)$ and bottom: pitch-rate tracking error $e_{Q}(t)$.

respect to realistically scaled disturbances. Specifically, a relative force disturbance is determined by comparing the drag force $D$ at Mach 8 at 85,000 ft (i.e., $7355 \mathrm{ft} / \mathrm{s}$ ) with the drag force after adding a $300 \mathrm{ft} / \mathrm{s}$ (e.g., a wind gust) disturbance. Using Newton's second law and dividing the drag force differential $\Delta D$ by the mass of the HSV $m$, a realistic upper bound for an acceleration disturbance $f_{\dot{V}}(t)$ was determined. Similarly, the same procedure can be performed, to compare the change in pitching moment $\Delta M$ caused by a $300 \mathrm{ft} / \mathrm{s}$ head wind gust. By dividing the moment differential by the moment of inertia of the HSV $I_{y y}$, a realistic upper bound for $f_{\dot{Q}}(t)$ can be determined. To calculate a reasonable angle-of-attack disturbance magnitude, a vertical wind gust of $300 \mathrm{ft} / \mathrm{s}$ is considered. By taking the inverse tangent of the vertical wind gust divided by the forward velocity at Mach 8 and 85,000 ft, an upper bound for the angle-ofattack disturbance $f_{\dot{\alpha}}(t)$ can be determined. Disturbances for the

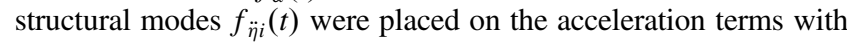
$\ddot{\eta}_{i}(t)$, where each subsequent mode is reduced by a factor of 10 relative to the first mode (see Fig. 7).

The proposed controller is designed to follow the outputs of a well behaved reference model. To obtain these outputs, a reference model that exhibited favorable characteristics was designed from a static linearized dynamics model of the full nonlinear dynamics [1]. The reference model outputs are shown in Fig. 8 . The velocity reference output follows a $1000 \mathrm{ft} / \mathrm{s}$ smooth step input, while the pitch rate performs several $\pm 1 \mathrm{deg} / \mathrm{s}$ maneuvers. The angle of attack stays within \pm 2 deg.
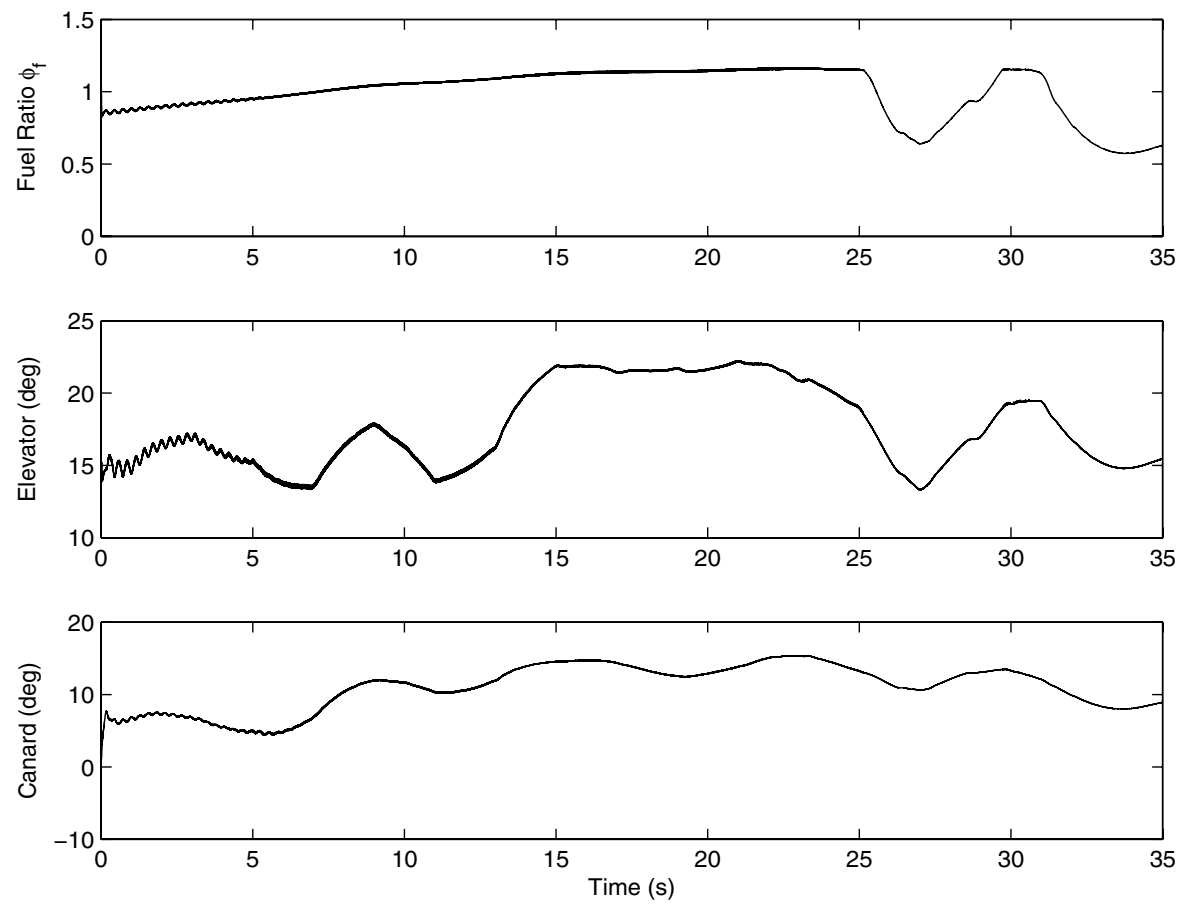

Fig. 12 Top: fuel equivalence ratio $\phi_{f}$, middle: elevator deflection $\delta_{e}$, and bottom: canard deflection $\delta_{c}$. 

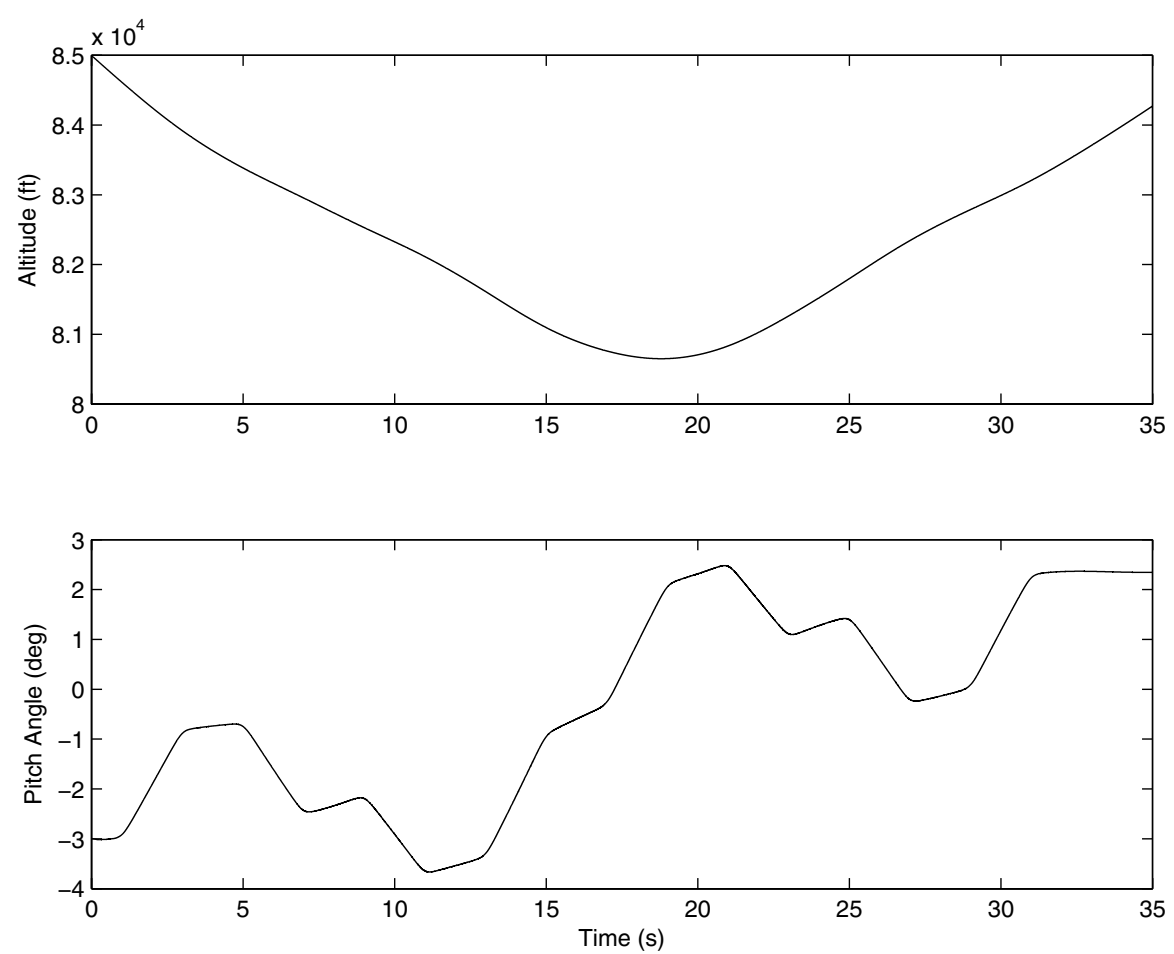

Fig. 13 Top: altitude $h(t)$ and bottom: pitch angle $\theta(t)$.

The control gains for Eqs. (15), (16), (25), and (26) are selected as

$$
\begin{gathered}
\gamma=10 \quad k_{s}=\operatorname{diag}\{5,1,300\} \quad k_{u}=\operatorname{diag}\{0.01,0.001,0.01\} \\
k_{\gamma}=\operatorname{diag}\{0.1,0.01,0.1\} \quad k_{\Gamma}=\operatorname{diag}\{1,0.5,1\}
\end{gathered}
$$

The control gains in Eq. (48) were obtained by choosing gains and then adjusting them based on the transient and steady-state performance. If the response exhibited a prolonged transient response (compared with the response obtained with other gains), the proportional gains were adjusted. If the response exhibited overshoot, derivative gains were adjusted. For this simulation, the control gains were tuned based on this trial-and-error basis. As a result of a conservative stability analysis, the final gains used may not satisfy the sufficient gain conditions developed in the control development and the theorem proof provided in the stability analysis. The subsequent results indicate that the developed controller can be applied despite the fact that some gain conditions may not be satisfied. In contrast to this trial-and-error approach, the control gains could have been adjusted using more methodical approaches as described in various survey papers on the topic $[30,31]$.

The $C$ matrix and knowledge of some nominal $B_{0}$ matrix must be known. The $C$ matrix is given by

$$
C=\left[\begin{array}{lllllllllll}
1 & 0 & 0 & 0 & 0 & 0 & 0 & 0 & 0 & 0 & 0 \\
0 & 1 & 0 & 0 & 0 & 0 & 0 & 0 & 0 & 0 & 0 \\
0 & 0 & 1 & 0 & 0 & 0 & 0 & 0 & 0 & 0 & 0
\end{array}\right]
$$
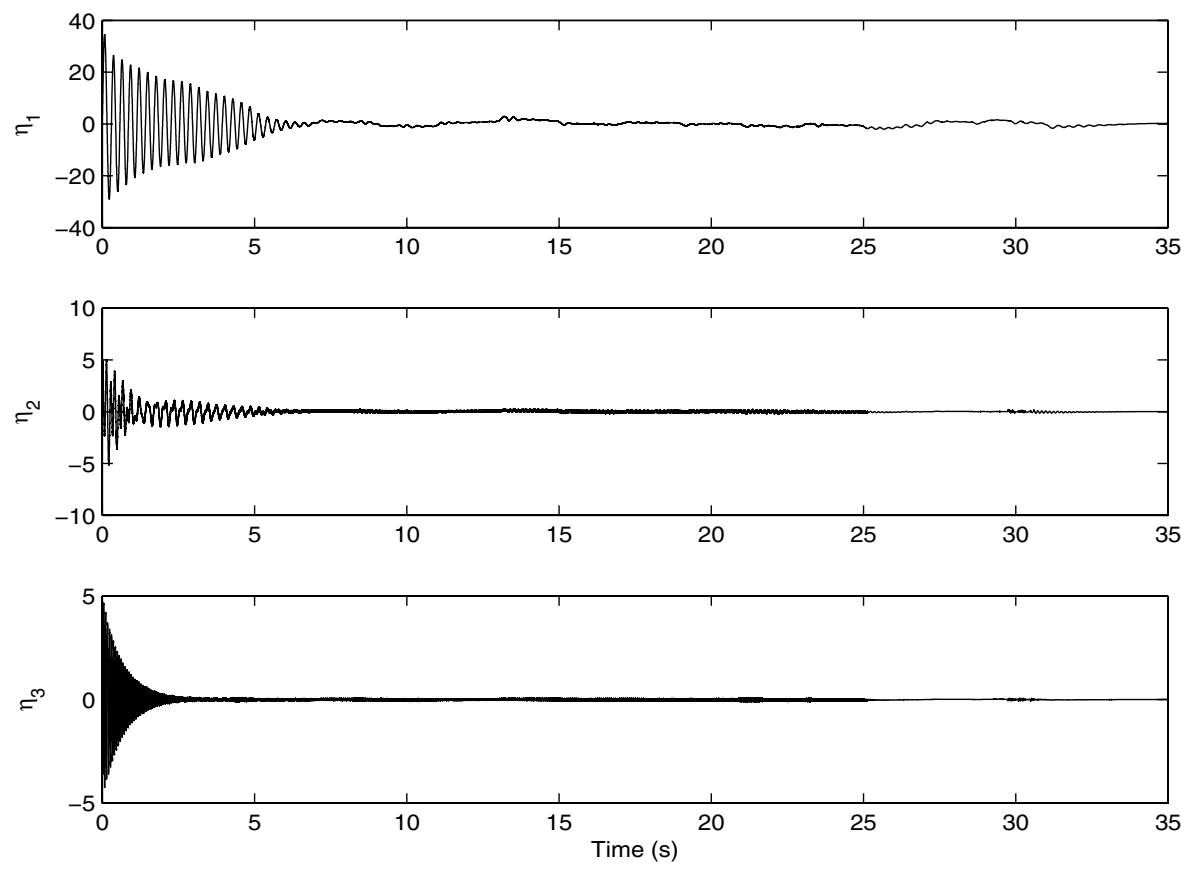

Fig. 14 Top: first structural elastic mode $\eta_{1}$, middle: second structural elastic mode $\eta_{2}$, and bottom: third structural elastic mode $\eta_{3}$. 
for the output vector of Eq. (9), and the $B_{0}$ matrix is selected as

$$
\begin{aligned}
& B_{0} \\
& =\left[\begin{array}{ccccccccccc}
-32.69 & -0.017 & -9.07 & 0 & 0 & 0 & 2367 & 0 & -1132 & 0 & -316 \\
25.72 & -0.0111 & 9.39 & 0 & 0 & 0 & 3189 & 0 & 2519 & 0 & 2067 \\
42.84 & -0.0016 & 0.0527 & 0 & 0 & 0 & 42.13 & 0 & 92.12 & 0 & -80.0
\end{array}\right]^{T}
\end{aligned}
$$

based on a linearized plant model about some nominal conditions.

The HSV has an initial velocity of Mach 7.5 at an altitude of $85,000 \mathrm{ft}$. The velocity, and velocity tracking errors are shown in Fig. 9. The angle of attack and angle-of-attack tracking error is shown in Fig. 10. The pitch rate and pitch tracking error are shown in Fig. 11. The control effort required to achieve these results is shown in Fig. 12. In addition to the output states, other states such as altitude and pitch angle are shown in Fig. 13. The structural modes are shown in Fig. 14.

\section{Conclusions}

This result represents the first ever application of a continuous, robust model reference control strategy for a hypersonic vehicle system with additive bounded disturbances and aerothermoelastic effects, where the control input is multiplied by an uncertain, column-deficient, parameter-varying matrix. A potential drawback of the result is that the control structure requires that the product of the output matrix with the nominal control matrix be invertible. For the output matrix and nominal matrix, the elevator and canard deflection angles and the fuel equivalence ratio can be used for tracking outputs such as the velocity, angle of attack, and pitch rate; or the velocity and flight-path angle; or the velocity, flight-path angle, and pitch rate. Yet, these controls cannot be applied to solve the altitude tracking problem because the altitude is not directly controllable and the product of the output matrix with the nominal control matrix is singular. However, the integrator backstepping approach that has been examined in other recent results for the hypersonic vehicle could potentially be incorporated in the control approach to address such objectives. A Lyapunov-based stability analysis is provided to verify the exponential tracking result. Although the controller was developed using a linear-parametervarying model of the hypersonic vehicle, simulations results for the full nonlinear model with temperature variations and exogenous disturbances illustrate the boundedness of the controller with favorable transient and steady-state tracking errors. These results indicate that the linear-parameter-varying model with exogenous disturbances is a reasonable approximation of the dynamics for the control development. However, due to the conservative nature of the robust Lyapunov-based design process, the sufficient gain conditions based on conservative bounding arguments do not provide a clear indication of how to select the control gains.

\section{Acknowledgment}

This research is supported in part by NASA NNX07AC46A with Program Manager Don Soloway.

\section{References}

[1] Bolender, M. A., and Doman, D. B., "Nonlinear Longitudinal Dynamical Model of an Air-Breathing Hypersonic Vehicle," Journal of Spacecraft and Rockets, Vol. 44, No. 2, 2007, pp. 374-387. doi:10.2514/1.23370

[2] Heeg, J., Zeiler, T. A., Pototzky, A. S., and Spain, C. V., "Aerothermoelastic Analysis of a NASP Demonstrator Model," AIAA Paper 93-1366, April 1993.

[3] Abbasa,L.K., Qian, C., Marzocca, P.,Zafer, G., and Mostafa, A., "Active Aerothermoelastic Control of Hypersonic Double-Wedge Lifting Surface," Chinese Journal of Aeronautics, Vol. 21, 2008, pp. 8-18. doi:10.1016/S1000-9361(08)60002-3

[4] Lind, R., "Linear Parameter-Varying Modeling and Control of Structural Dynamics with Aerothermoelastic Effects," Journal of Guidance, Control, and Dynamics, Vol. 25, No. 4, July-Aug. 2002, pp. 733-739.

doi: $10.2514 / 2.4940$

[5] Marrison, C. I., and Stengel, R. F., "Design of Robust Control Systems for a Hypersonic Aircraft," Journal of Guidance, Control, and Dynamics, Vol. 21, No. 1, 1998, pp. 58-63. doi: $10.2514 / 2.4197$

[6] Wang, Q., and Stengel, R. F., "Robust Nonlinear Control of a Hypersonic Aircraft," Journal of Guidance, Control, and Dynamics, Vol. 23, No. 4, 2000, pp. 577-585.

doi: $10.2514 / 2.4580$

[7] Austin, K. J., and Jacobs, P. A., "Application of Genetic Algorithms to Hypersonic Flight Control," IFSA World Congress and 20th NAFIPS International Conference, Inst. of Electrical and Electronics Engineers, Piscataway, NJ, July 2001, pp. 2428-2433.

[8] Miyasato, Y., "Adaptive Gain-Scheduled H-infinity Control of Linear Parameter-Varying Systems with Nonlinear Components," American Control Conference, Inst. of Electrical and Electronics Engineers, Piscataway, NJ, June 2003, pp. 208-213. doi:10.1109/ACC.2003.1238939

[9] Parker, J. T., Serrani, A., Yurkovich, S., Bolender, M. A., and Doman, D. B., "Control-Oriented Modeling of an Airbreathing Hypersonic Vehicle," Journal of Guidance, Control, and Dynamics, Vol. 30, No. 3, 2007, pp. 856-869. doi: $10.2514 / 1.27830$

[10] Bolender, M., and Doman, D., "A Non-Linear Model for the Longitudinal Dynamics of a Hypersonic Air-Breathing Vehicle," AIAA Paper 2005-6255, Aug. 2005.

[11] Sigthorsson, D., Jankovsky, P., Serrani, A., Yurkovich, S., Bolender, M., and Doman., D., "Robust Linear Output Feedback Control of an Air-Breathing Hypersonic Vehicle," Journal of Guidance, Control, and Dynamics, Vol. 31, No. 4, July 2008, pp. 1052-1066. doi: $10.2514 / 1.32300$

[12] Fiorentini, L., Serrani, A., Bolender, M. A., and Doman, D. B., "Nonlinear Robust Adaptive Control of Flexible Air-Breathing Hypersonic Vehicle," Journal of Guidance, Control, and Dynamics, Vol. 32, No. 2, April 2009, pp. 402-417. doi: $10.2514 / 1.39210$

[13] Gibson, T., Crespo, L., and Annaswamy, A., "Adaptive Control of Hypersonic Vehicles in the Presence of Modeling Uncertainties," American Control Conference, Inst. of Electrical and Electronics Engineers, Piscataway, NJ, June 2009, pp. 3178-3183. doi:10.1109/ACC.2009.5160746

[14] Fiorentini, L., Serrani, A., Bolender, M., and Doman, D., "Nonlinear Control of Non-Minimum Phase Hypersonic Vehicle Models," American Control Conference, Inst. of Electrical and Electronics Engineers, Piscataway, NJ, June 2009, pp. 3160-3165. doi:10.1109/ACC.2009.5160211

[15] Serrani, A., Zinnecker, A., Fiorentini, L., Bolender, M., and Doman, D. "Integrated Adaptive Guidance and Control of Constrained Nonlinear Air-Breathing Hypersonic Vehicle Models," American Control Conference, Inst. of Electrical and Electronics Engineers, Piscataway, NJ, June 2009, pp. 3172-3177. doi:10.1109/ACC.2009.5160694

[16] Williams, T., Bolender, M. A., Doman, D. B., and Morataya, O., "An Aerothermal Flexible Mode Analysis of a Hypersonic Vehicle," AIAA Paper 2006-6647, Aug. 2006.

[17] Culler, A. J., Williams, T., and Bolender, M. A., "Aerothermal Modeling and Dynamic Analysis of a Hypersonic Vehicle," AIAA Atmospheric Flight Mechanics Conference, Hilton Head, SC, AIAA Paper 2007-6395, Aug. 2007.

[18] Bolender, M., and Doman, D., "Modeling Unsteady Heating Effects on the Structural Dynamics of a Hypersonic Vehicle," AIAA Paper 20066646, Aug. 2006.

[19] Vosteen, L. F., "Effect of Temperature on Dynamic Modulus of Elasticity of Some Structural Allows," NASA Langley Research Center, Aeronautical Lab., TR 4348, Hampton, VA, Aug. 1958.

[20] Bhat, S., and Lind, R., "Control-Oriented Analysis of Thermal Gradients for a Hypersonic Vehicle," American Control Conference, Inst. of Electrical and Electronics Engineers, Piscataway, NJ, 2009, pp. 2513 2518. doi:10.1109/ACC.2009.5160180

[21] Wilcox, Z. D., MacKunis, W., Bhat, S., Lind, R., and Dixon, W. E., "Robust Nonlinear Control of a Hypersonic Aircraft in the Presence of Aerothermoelastic Effects," American Control Conference, Inst. of Electrical and Electronics Engineers, Piscataway, NJ, June 2009, pp. 2533-2538. doi:10.1109/ACC.2009.5160480

[22] Patre, P. M., MacKunis, W., Makkar, C., and Dixon, W. E., "Asymptotic Tracking for Systems with Structured and Unstructured Uncertainties," 
IEEE Transactions on Control Systems Technology, Vol. 16, No. 2, 2008, pp. 373-379. doi:10.1109/TCST.2007.908227

[23] Xian, B., Dawson, D. M., de Queiroz, M. S., and Chen, J., "A Continuous Asymptotic Tracking Control Strategy for Uncertain Nonlinear Systems," IEEE Transactions on Automatic Control, Vol. 49, No. 7, July 2004, pp. 1206-1211. doi:10.1109/TAC.2004.831148

[24] Fillipov, A. F., Differential Equations with Discontinuous Righthand Sides, Kluwer Academic, Norwell, MA, 1988, pp. 48-122.

[25] Gutman, S., "Uncertain Dynamical Systems-A Lyapunov Min-Max Approach," IEEE Transactions on Automatic Control, Vol. 24, No. 3, 1979, pp. 437-443. doi:10.1109/TAC.1979.1102073

[26] Patre, P., Mackunis, W., Johnson, M., and Dixon, W., "Composite Adaptive Control for Euler-Lagrange Systems with Additive Disturbances," Automatica, Vol. 46, No. 1, 2010, pp. 140-147. doi:10.1016/j.automatica.2009.10.017

[27] Patre, P. M., Mackunis, W., Makkar, C., and Dixon, W. E., "Asymptotic Tracking for Systems with Structured and Unstructured Uncertainties,"
IEEE Transactions on Control Systems Technology, Vol. 16, No. 2, 2008, pp. 373-379. doi:10.1109/TCST.2007.908227

[28] Patre, P. M., MacKunis, W., Kaiser, K., and Dixon, W. E., "Asymptotic Tracking for Uncertain Dynamic Systems via a Multilayer Neural Network Feedforward and RISE Feedback Control Structure," IEEE Transactions on Automatic Control, Vol. 53, No. 9, 2008, pp. 21802185. doi:10.1109/TAC.2008.930200

[29] Qu, Z., and Xu, J. X., "Model-Based Learning Controls and their Comparisons Using Lyapunov Direct Method," Asian Journal of Control, Vol. 4, No. 1, Mar. 2002, pp. 99-110.

[30] Killingsworth, N. J., and Krstic, M., "PID Tuning Using Extremum Seeking," IEEE Control Systems Magazine, Vol. 26, No. 1, Feb. 2006, pp. 70-79. doi:10.1109/MCS.2006.1580155

[31] Åström, K. J., Hägglund, T., Hang, C. C., and Ho, W. K., "Automatic Tuning and Adaptation for PID Controllers-A Survey," Control Engineering Practice, Vol. 1, No. 4, 1993, pp. 669-714. doi:10.1016/0967-0661(93)91394-C 